Proc. Indian Acad. Sci. (Chem. Sci.), Vol. 105, No. 6, December 1993, pp. 773-782.

(C) Printed in India.

\title{
Resonant two-photon ionization processes of van der Waals adducts: Spectroscopy and reactivity of styrenes clustered with various molecules
}

\author{
A GIARDINI-GUIDONI ${ }^{\mathrm{a}}$, A MELE ${ }^{\mathrm{a}}, \mathrm{S}$ PICCIRILLO ${ }^{\mathrm{a}}$, M CORENO ${ }^{\mathrm{a}}$, \\ and M SNELS ${ }^{\mathrm{b} *}$ \\ ${ }^{21}$ Universitá degli Studi di Roma "La Sapienza", Dipartimento di Chimica, P le A Moro, \\ 5, I-00185 Rome, Italy

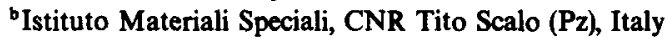

\begin{abstract}
Research on the formation and properties of clusters of aromatic molecules bonded to numerous solvents is rapidly expanding. Recently much attention has been paid to these adduct species with the objective of clarifying nucleation phenomena. Photophysical and photochemical studies of these clusters give information on solvent effects, intracluster reactions and charge-transfer processes. Model calculations of potential surfaces of vdW adducts have provided knowledge of the intracluster binding energy and vdW vibrational modes in a few systems. Here, data are reported on resonant two-photon ionization R2PI mass spectra and on spectroscopic shifts of styrenes clustered with various atoms and
\end{abstract} \\ molecules.
}

Keywords. Two-photon ionization; van der Waals adducts; styrene clusters.

\section{Introduction}

The importance of van der Waals ( $\mathrm{vdW}$ ) interactions in the study of aromatic systems bonded to solvent molecules has been amply recognized (Bernstein 1990; Even et al 1990). Studies on clusters of these compounds by reactive and unreactive moieties are particularly valuable in understanding condensation and nucleation phenomena, energetics of inter- and intramolecular modes and finally kinetics of reactive processes (Brutschy 1990; Keese and Castleman 1990).

Production of these adducts can be achieved by adiabatic expansion in vacuum through a nozzle of solute-solvent mixtures. By varying the pressure and temperature at the nozzle, it is possible to change the size distribution of clusters formed in the expansion. The clusters are usually analysed by laser-induced fluorescence (LIF), IR spectroscopy or mass spectrometry. Resonance enhanced two-photon ionization (R2PI) combined with time-of-flight (TOF) detection of the adducts has been demonstrated to be a powerful technique suitable for both spectroscopy and reactivity studies (Breen et al 1989; Brutschy 1990).

\footnotetext{
* For correspondence
} 
Spectroscopic studies allow us to probe the solvent environment and how it affects the solute electronic structure. In particular, the evolution of the solute absorption spectrum, as a function of the increasing number of solvent molecules, can be related to a coalescence process leading to the condensed phase. Another important aspect is the identification through mass spectrometric analysis of the solute-solvent intracluster reaction which can take place within a very short time after ionization.

In this paper, results are reported on the spectroscopy and reaction dynamics of styrene (STY) and 4-fluorostyrene (4FSTY) clustered with noble gases and several substances. These aromatic molecules are sensitive probes of the molecular interaction with solvent media. Their choice has been dictated also by the fact that the first excited state $S_{1}$ is for both approximately half way with respect to the ionization continuum. The cluster ions produced in the R2PI process have little excess energy and it may be assumed that fragmentation plays a minor role. A comparison between the spectroscopy and the reactivity of these two systems, with respect to the solvent molecules, is reported here.

\section{Experimental methods}

The experimental apparatus used to produce supersonic molecular beams of clusters, shown in figure 1 together with the ionization laser and TOF mass spectrometer, has been already described (Giardini Guidoni et al 1993) and will be only briefly summarized here.

The system consists of two independently pumped vacuum chambers, separated by a skimmer of $1 \mathrm{~mm}$ inner diameter. The clusters are formed in a supersonic expansion of $\mathrm{He}$, seeded with the aromatic system, either pure or mixed with solvent molecules in a suitable ratio. The stagnation pressure can be varied between 0.5 and

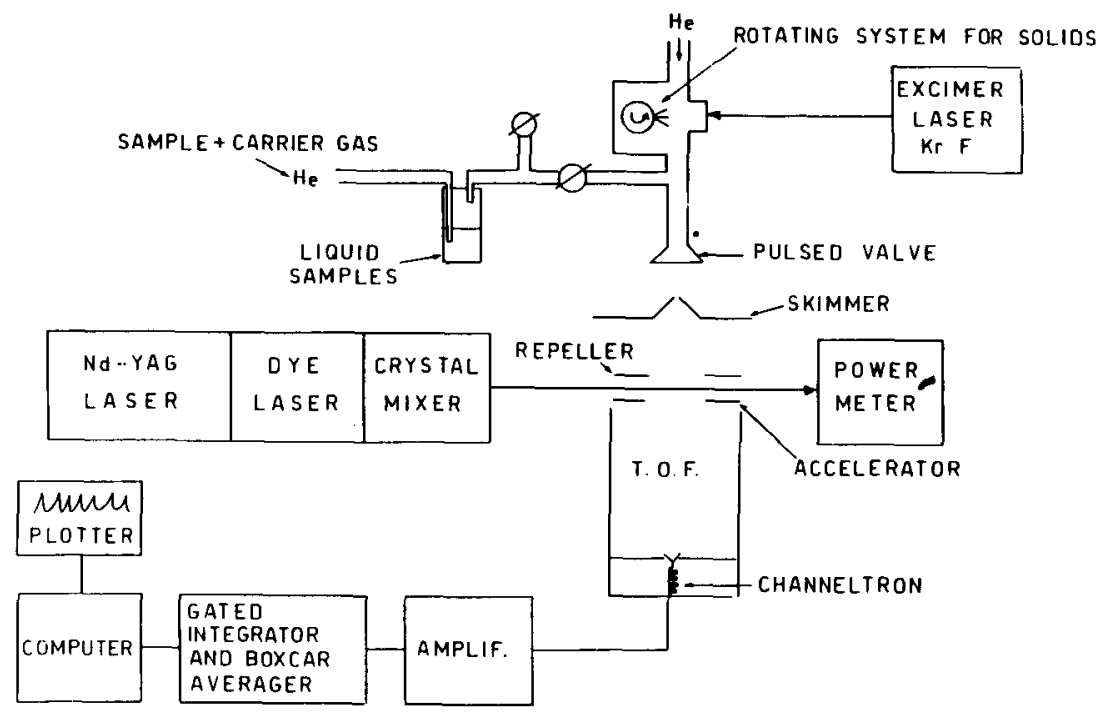

Figure 1. Schematic of the experimental apparatus for the production and detection of clusters. 
4 bar. The expansion in the first chamber is obtained through a pulsed valve (General Valve) with a nozzle of $400 \mu \mathrm{m}$ inner diameter and aperture time up to $600 \mu \mathrm{s}$. The repetition time is $10 \mathrm{~Hz}$. The pressure in the first chamber is kept around $10^{-5}$ torr. The skimmed beam is crossed perpendicularly by the ionization laser $30 \mathrm{~cm}$ downstream between the first two acceleration plates of a Wiley-McLaren TOF mass spectrometer. The resolving power of the TOF is 1 mass unit at $m / e 200$. The clusters are ionized by one colour R2PI process by using a frequency doubled dye laser pumped by an Nd: YAG (Quantel model 710). The relative intensity of the ion signal depends on the expansion conditions: stagnation pressure, time lag between the dye laser pulse relative to the gas pulse and laser wavelength.

The compounds studied are STY and 4FSTY, pure or mixed with $\mathrm{Ar}, \mathrm{CCl}_{4}, \mathrm{NH}_{3}$. $\mathrm{C}_{2} \mathrm{H}_{5} \mathrm{NH}_{2}$ (MEA) and $\left(\mathrm{C}_{2} \mathrm{H}_{5}\right)_{2} \mathrm{NH}$ (DEA). STY and 4FSTY are used as supplied by Aldrich Chemicals without any further purification.

\section{Spectroscopy}

Spectroscopy of STY and 4FSTY, either pure or bound to reactive or non-reactive systems, deals mainly with $0_{0}^{\circ}$ transitions from the ground state $S_{0}$ to the lowest singlet excited state $S_{1}$. The spectral shift near the vibronic origin of this $\pi \rightarrow \pi^{*}$ transition occurs in various chromophore solvent adducts as largely reported in the literature (Bernstein 1990, and references therein). The spectral shift of a given electronic transition with respect to the isolated molecule is a measure of the relative energy difference between the lower and upper electronic states induced by aggregation. The magnitude and direction of the electronic shift is strongly related to the nature of the interaction between the chromophore which is the solute and the solvent. Of course, a red shift indicates a smaller energy difference between the ground and the excited states in the adduct, a blue shift a larger difference.

The spectra of STY.Ar $r_{n}$ clusters are reported in figure 2, together with the spectrum of pure STY. As shown, the structure of STY.Ar and STY'Ar ${ }_{2}$ have sharp origins and exhibit features due to vdW modes. The STY.Ar $0_{0}^{0}$ transition is shifted by $32 \mathrm{~cm}^{-1}$ with respect to pure STY spectrum, confirming previous measurements (Dimopoulou-Rademann et al 1988). The STY.Ar ${ }_{2}$ spectral shift is $63 \mathrm{~cm}^{-1}$, almost twice that of STY·Ar. This behaviour is analogous to what was found for other aromatic systems (Bernstein 1990). It is explained by the presence of two equivalent sites for the Ar atoms, one above and the other below the aromatic ring. The STY.Ar exhibits complex features due to the more numerous intramolecular vdW modes and eventually to the presence of isomeric structures (Hermine et al 1992). Analogous data have been obtained in the case of 4FSTY-Ar clusters (Piccirillo et al 1993).

Spectra of some STY $\left(\mathrm{CCl}_{4}\right)_{n}$ complexes are reported in figure 3 . The red shift for the $0_{0}^{0}$ transition of the STY.CCl 4 adduct is $89 \mathrm{~cm}^{-1}$, much larger than that found for STY'Ar. This fact can be explained by the much larger polarizability of the $\mathrm{CCl}_{4}$ molecule with respect to Ar. As the cluster size increases to $n$ values larger than 2 , both broad and sharp components are observed in the spectra. The shift of the band maxima as a function of the $n$ solvent molecules shows an evolution toward the red with respect to the $0_{0}^{\circ}$ transition of the bare STY molecule. The large bandwidth can be attributed to the presence of interaction between the chromophore and the solvent molecules leading to the formation of different isomers (Even et al 1990). The UV 


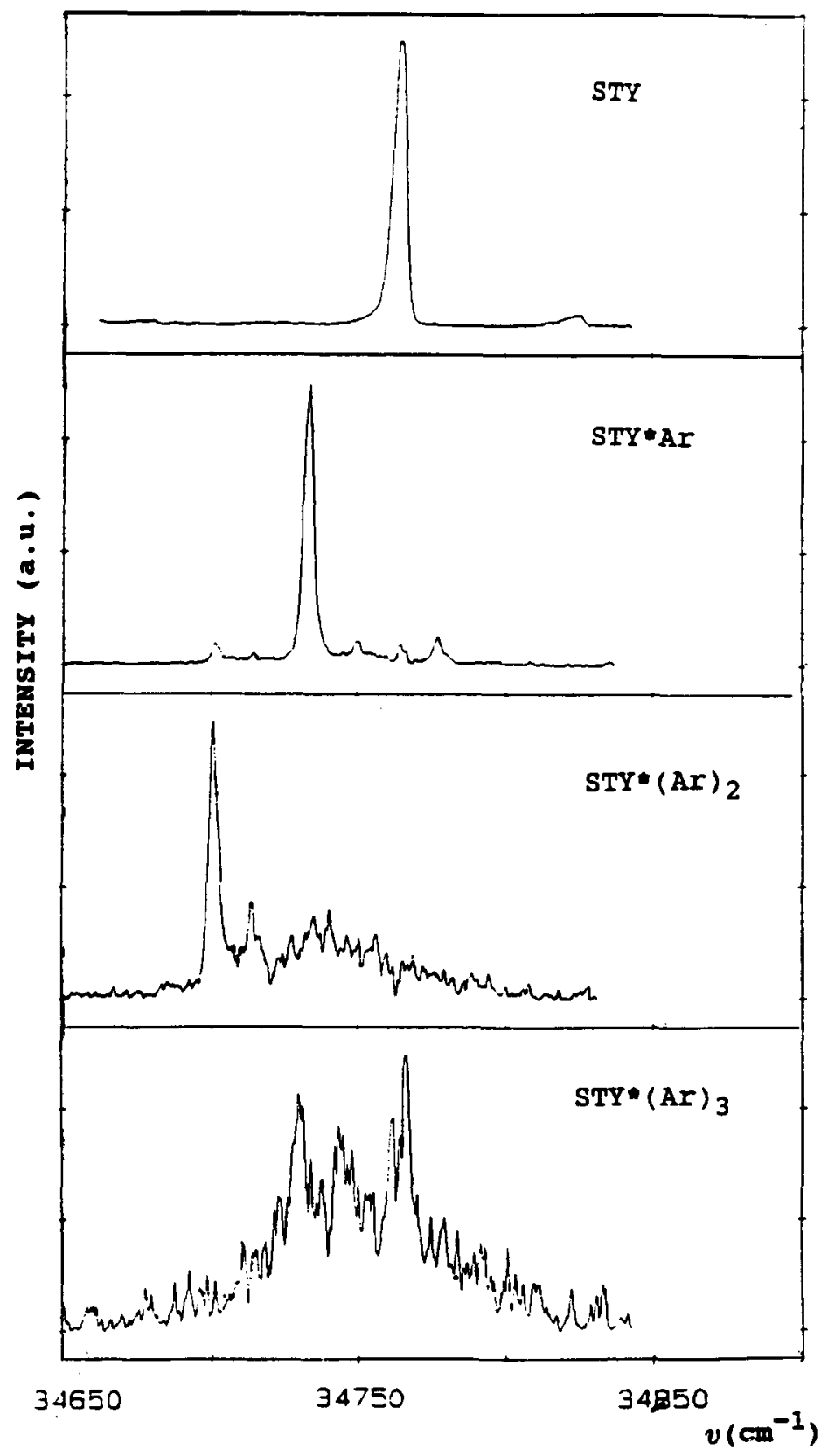

Figure 2. The mass resolved R2PI spectra of styrene clustered by Ar atoms. The spectral shift scale is relative to the $S_{1}$ electronic origin of styrene.

absorption band of STY in $\mathrm{CCl}_{4}$ solution (insert in figure 3) has a maximum centred at $34,180 \mathrm{~cm}^{-1}$. The resulting red shift of $580 \mathrm{~cm}^{-1}$ with respect to pure STY in the gas phase can be the tendencial value of STY. $\left(\mathrm{CCl}_{4}\right)_{n}$ for very large $n$, indicating that these aggregates represent the first building blocks of a solute molecule in $\mathrm{CCl}_{4}$ solution. 


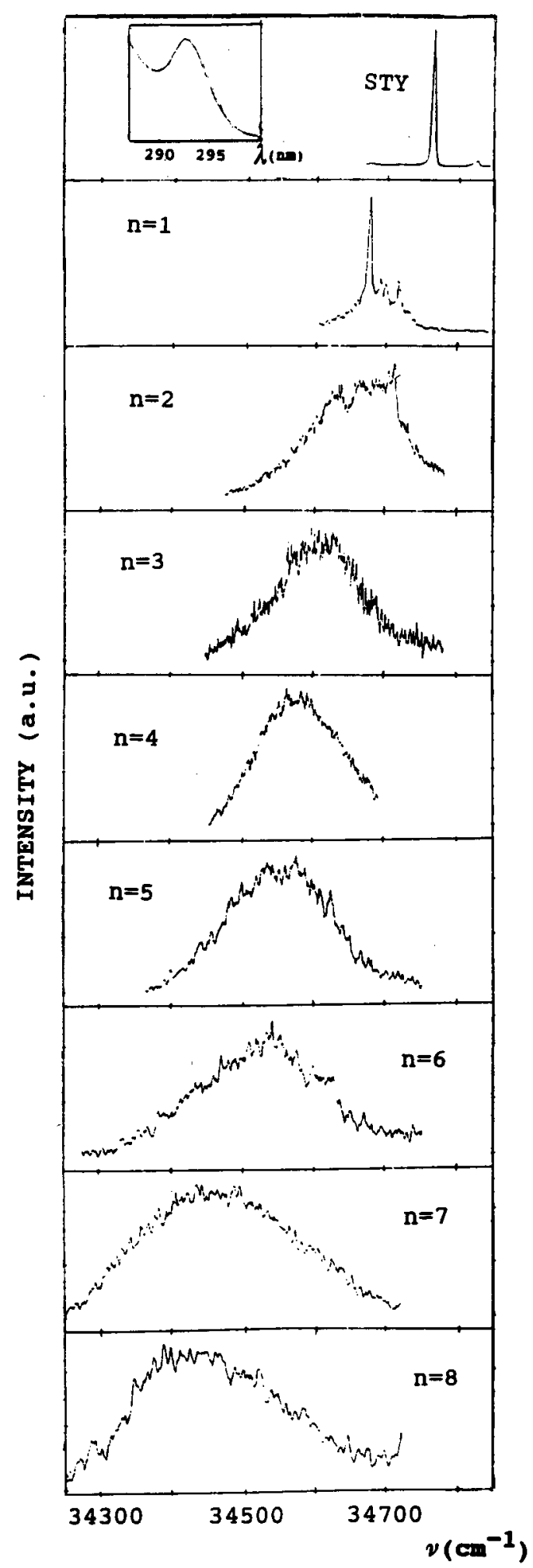

Figure 3. The mass resolved $\mathrm{R} 2 \mathrm{PI}$ spectra of styrene clustered by $\mathrm{CCl}_{4}$ molecules. The spectral shift scale is relative to the $S_{1}$ electronic origin of styrene. 


\section{Reactivity}

It is well known that, following R2PI ionization, reactions can take place within the cluster. Resonant two-photon ionization can lead to intracluster processes where the chromophore solute reacts with the solvent either by nucleophilic substitution or by charge or proton transfer reaction (Breen et al 1989; Brutschy 1990).

The following scheme represents possible intracluster solvent (S) and chromophore (C) reactions:

$$
\text { Proton transfer } \quad(\mathrm{C}-\mathrm{H}) \mathrm{S}_{m-n}+\mathrm{S}_{n} \mathrm{H}^{+},
$$

$$
\left(\mathrm{C} \mathrm{S}_{m}\right)^{+}
$$

Charge transfer $\quad \mathrm{C} \mathrm{S}_{m-n}+\mathrm{S}_{n}^{+}$.

Cluster ions $\mathrm{S}_{n}^{+}$from solvent molecules having a dual character may lead to autoprotonation:

$$
\mathrm{S}_{n}^{+} \quad \text { autoprotonation } \quad \mathrm{S}_{n-l}(\mathrm{~S}-\mathrm{H})+\mathrm{S}_{l-1} \mathrm{H}^{+},
$$

where $\mathrm{C}-\mathrm{H}$ and $\mathrm{S}-\mathrm{H}$ stands for an $\mathrm{H}$ lost.

In styrenes complexed with amines we have found the occurrence of internal ion-molecule reactions which lead to the formation of protonated species. Typical
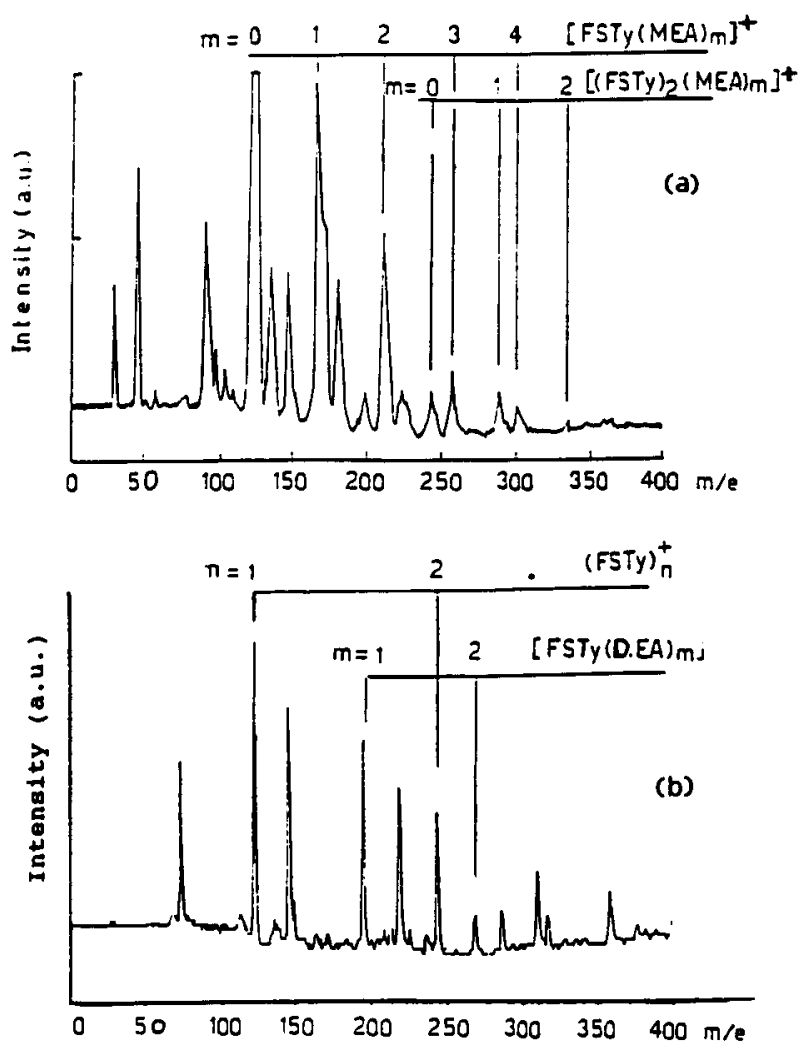

Figure 4. Mass spectra of a mixture of 4FSTY with MEA (a) and DEA (b), taken at a laser frequency of $34,350 \mathrm{~cm}^{-1}(292 \mathrm{~nm})$. 


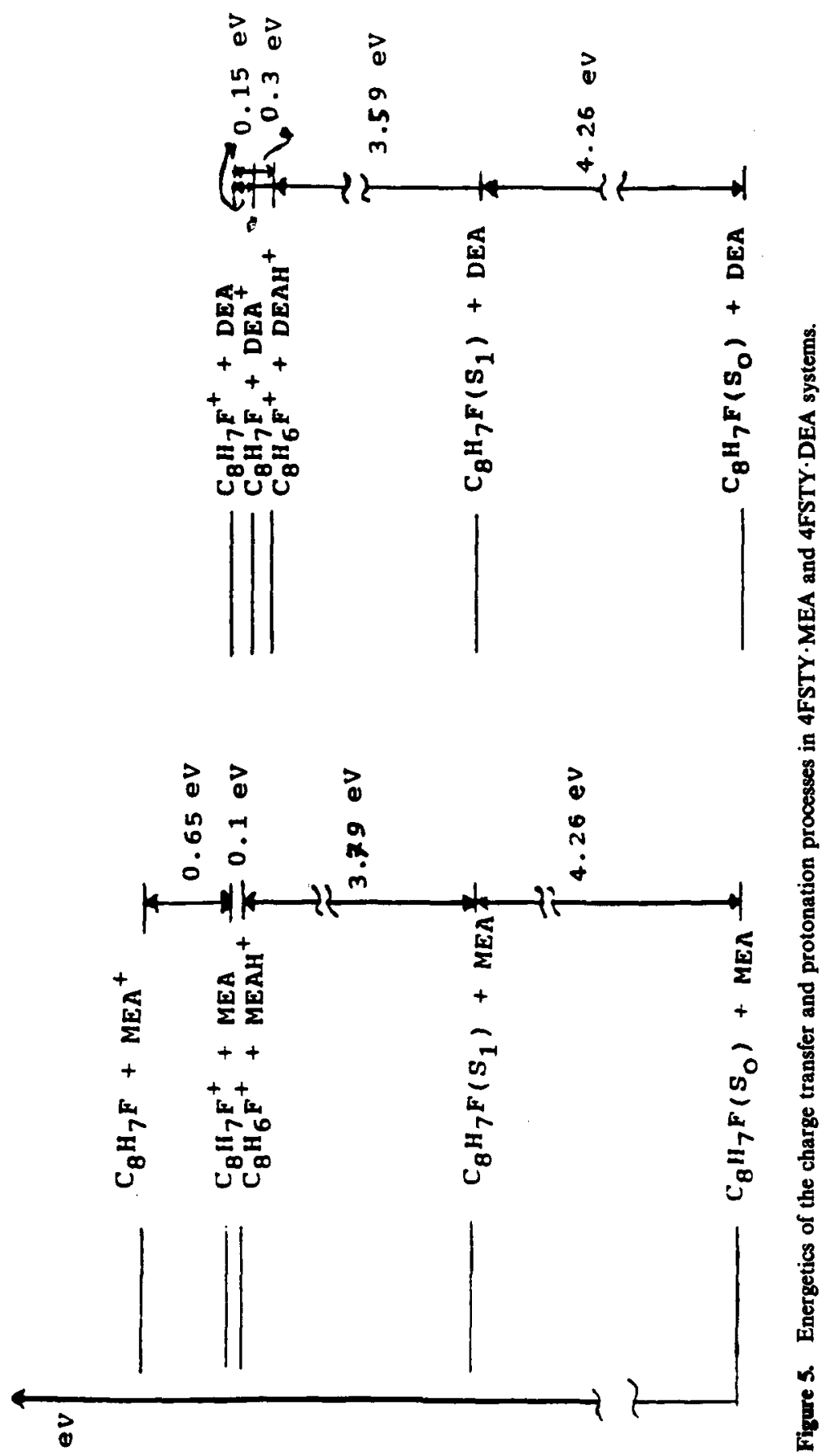


mass spectra of 4 FSTY.MEA and 4FSTY.DEA taken at $34,250 \mathrm{~cm}^{-1}$ are reported in figure 4.

In the spectra of 4FSTY and MEA mixtures (figure 4a), besides the main cluster sequences, $4 \mathrm{FSTY}(\mathrm{MEA})_{m}^{+}$and $(4 \mathrm{FSTY})_{2} \cdot(\mathrm{MEA})_{m^{\prime}}^{+}$several peaks can be observed.

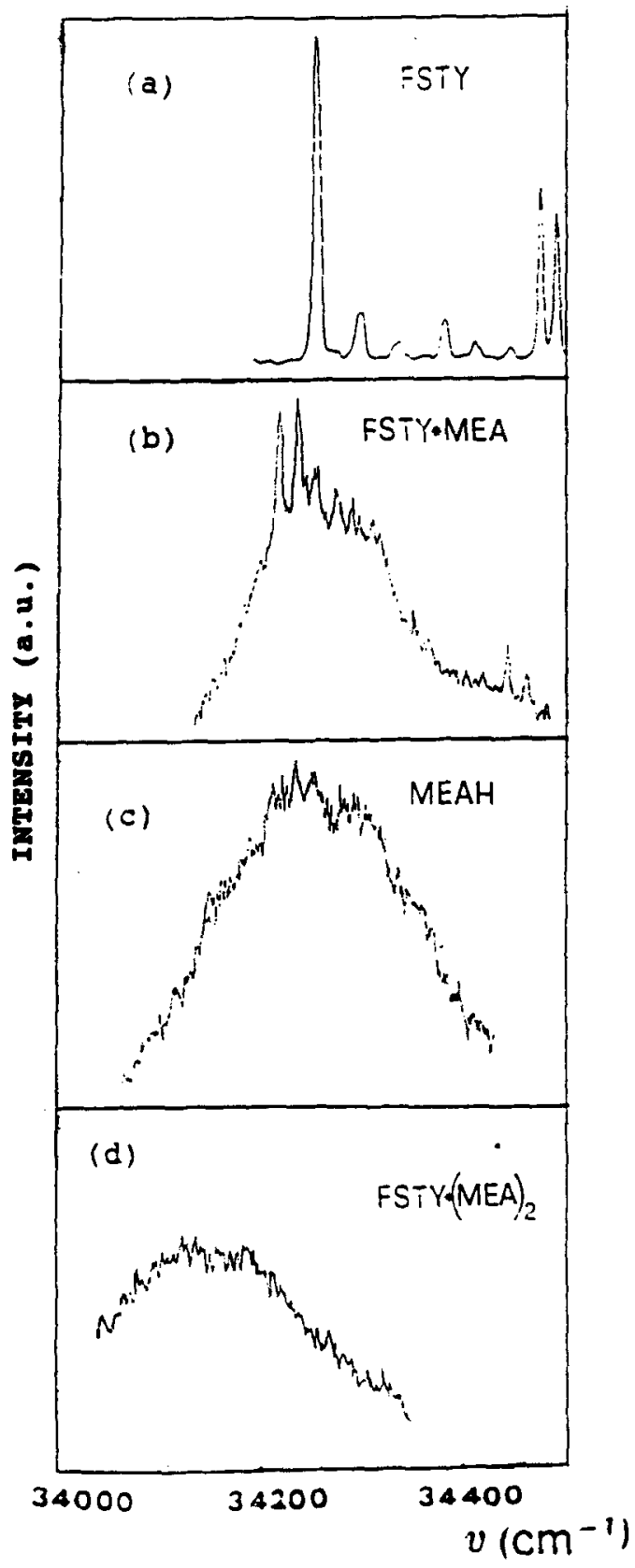

Figure 6. Wavelength dependence of $4 \mathrm{FSTY}{ }^{+}(\mathrm{a}),(4 \mathrm{FSTY} \cdot \mathrm{MEA})^{+}(\mathrm{b}), \mathrm{MEAH}^{+}$(c) and $\left[4 \mathrm{FSTY} \cdot(\mathrm{MEA})_{2}\right]^{+}$(d). 
These peaks have been assigned to $(\mathrm{MEA})_{m}^{+}, \mathrm{MEAH}^{+}$and protonated (MEA) $\mathrm{H}^{+}$ clusters. Other peaks attributed to fragmentation are also observed. 4FSTY and DEA mixtures (figure $4 \mathrm{~b}$ ) behave similarly and the spectra are characterized by main cluster sequences. Other peaks have been assigned to intracluster charge transfer products. Addition of $\mathrm{NH}_{3}$ to the 4FSTY beam also produces cluster sequences but only at very high ratios of $\mathrm{NH}_{3} / 4 \mathrm{FSTY}$ are intracluster reactions observed.

With reference to the transfer reaction scheme, a diagram of the energetics has been plotted in figure 5 for 4FSTY with MEA, and DEA, using literature data (Lias et al 1984; Brutschy 1990) and not accounting for the cluster binding energy.

From the energetics values reported in figure 5 , the proton transfer reaction in the MEA adduct appears to be slightly exothermic $(0 \cdot 1 \mathrm{eV})$ and the charge transfer process endothermic by about $0.65 \mathrm{eV}$. This fact supports the hypothesis that $\mathrm{MEAH}^{+}$is formed mainly by direct intracluster proton transfer from 4FSTY to solvent. The data obtained on a single mass selected in the TOF spectrum as a function of laser frequency are reported in figure 6 (c) at $m / e 46$ for $\mathrm{MEAH}^{+}$, (a) at $m / e 122$ for $\mathrm{FSTY}^{+}$, (b) at $m / e 167$ for $(4 \mathrm{FSTY} \cdot \mathrm{MEA})^{+}$and (d) at $m / e 212$ for $\left(4 \mathrm{FSTY} \cdot(\mathrm{MEA})_{2}\right)^{+}$. The wavelength dependence of $\mathrm{MEAH}^{+}$, though less structured, has the same trend of 4FSTY-MEA ${ }^{+}$. This confirms the hypothesis that the former ion is a product of the direct intracluster proton transfer (1). It can be noted that the absorption spectrum of 4FSTY is characterized by sharp peaks attributed to the vibronic levels of the $S_{1} \leftarrow S_{0}$ electronic transition. Wavelength excitation of the 4FSTY'MEA cluster shows, on the top of a broad continuum, a fine structure related to $\mathrm{vdW}$ and vibronic modes. Some intensity contribution towards the red of the wavelength in $\mathrm{MEAH}^{+}$spectrum is observed. This feature may arise from the contribution of other processes yielding $\mathrm{MEAH}^{+}$. In particular, dissociative charge transfer followed by autoprotonation (3) of higher cluster ions $\left[4 \mathrm{FSTY} \cdot(\mathrm{MEA})_{m}\right]^{+}$should be taken into account. Typically 4FSTY.(MEA $)_{2}$ absorption is shifted further toward the red with respect to pure 4FSTY (figure 6). This would justify a contribution to the spectrum of $\mathrm{MEAH}^{+}$in the red region.

In the case of the 4FSTY-DEA adduct, both charge and proton transfer reactions are exothermic by 0.15 and $0.3 \mathrm{eV}$, respectively. As already hypothesized (Brutschy 1990; Keese and Castleman 1990), if energetically allowed, charge transfer processes always dominate other competing processes. In the TOF mass spectrum, peaks corresponding to (DEA) ${ }_{n}^{+}$cluster ions appear to be asymmetrically broadened; the presence of this tail can be ascribed to the proton transfer pathway leading to $(\mathrm{DEA})_{n} \mathrm{H}^{+}$formation.

\section{Acknowledgements}

This work is partly supported by the National Research Council (CNR) of Italy under Progetto Finalizzato "Electrooptical Technologies" and "Chimica Fine".

\section{References}

Bernstein E R (ed.) 1990 In Atomic and molecular clusters (Amsterdam: Elsevier) p. 551

Breen J J, Kilgore K, Tzeng W B, Keese R G and Castleman A W Jr 1989 J. Chem. Phys. 9011

Brutschy B 1990 J. Phys. Chem. 948637 
Dimopoulou-Rademann O, Even U, Amirav A and Jortner J 1988 J. Phys. Chem. 925371

Even U, Ben Horin N and Jortner J $1990 \mathrm{~J}$. Chem. Phys. Lett. 150138

Jiardini Guidoni A, Piccirillo S, Coreno M, Snels M, Morone A and Teghil R 1993 Appl. Surf. Sci. 69340

Hermine P, Coutant B, Amar F G and Brechignac Ph 1992 Z. Phys. D22 529

Keese R G and Castleman A W Jr 1990 In Atomic and molecular clusters (ed.) E R Bernstein (Amsterdam: Elsevier) p. 507

jias S G, Liebman J F and Levin R D 1984 J. Phys. Chem. Ref. Data 13695

'iccirillo S, Coreno M, Giardini Guidoni A, Pizzella G, Snels M and Teghil R 1993 J. Mol. Struct. (to be published) 